\title{
The Influence of Macrophage-Activating Lipopeptide 2 (MALP-2) on Local and Systemic Inflammatory Response in a Murine Two-Hit Model of Hemorrhagic Shock and Subsequent Sepsis
}

\author{
Ding Xu $\oplus^{1,2,5}$ Klemens Horst, ${ }^{1}$ Weikang Wang, ${ }^{1}$ Peng Luo, ${ }^{1,3}$ Yulong Shi, ${ }^{1}$ Thomas Tschernig, ${ }^{4}$ \\ Johannes Greven, ${ }^{1}$ and Frank Hildebrand ${ }^{1}$
}

(Received May 3, 2020; accepted August 19, 2020)

Abstract - Pulmonary complications after severe trauma and sepsis remain to be the main cause for adverse outcome. MALP-2 has been described to exert beneficial effects on organ damage and the further course after isolated trauma and sepsis. However, the impact of MALP-2 on a clinically realistic two-hit scenario of trauma and subsequent sepsis remains unknown. We, therefore, investigated if the systemic inflammatory response and pulmonary immune response and damage are beneficially modulated by MALP-2 in a murine two-hit model. Blood pressure-controlled trauma-hemorrhage $(\mathrm{TH})$ and cecal ligation and puncture (CLP) were induced in C57/BL6 mice. Mice were divided into 2 control groups (control 1: TH without CLP; control 2: TH and CLP) and 3 experimental groups treated with MALP-2 at different time points (ETH, end of TH; ECLP, end of CLP; and 6CLP $6 \mathrm{~h}$ after CLP). Survival rates were assessed over the observation period of $168 \mathrm{~h}$ after the induction of TH. Concentrations of plasma inflammatory cytokines and chemokines (TNF- $\alpha$, IL- 6, MIP-1 $\alpha$, IFN- $\gamma$, and IL-10) were assessed, and bacterial clearance of the lungs was determined. Furthermore, pulmonary MPO activity assay to evaluate the infiltration of polymorphonuclear neutrophils $(\mathrm{PMN})$ and histological evaluation were performed. Survival rates were evaluated. Compared with control group 1, the level of TNF- $\alpha$ in the ECLP group showed a significant increase (ECLP, 2.27 pg. $/ \mathrm{ml} \pm 1.39 v s$. control 1: 0.16 pg./ml $\pm 0.11, p=0.021$ ). In contrast, levels of IFN- $\gamma$ were significantly reduced in groups ETH and 6CLP compared with control group 1 (control 1: 8.92 pg. $/ \mathrm{ml} \pm 4.38$ vs. ETH: 1.77 pg./ml $\pm 4.34, p=0.026$ resp. vs. 6CLP: 1.83 pg./ $\mathrm{ml} \pm 4.49, p=0.014)$. While systemic concentrations of inflammatory mediators were not

Ding $\mathrm{Xu}$ and Klemens Horst contributed equally to this work.

${ }^{1}$ Department of Orthopedic Trauma Surgery, RWTH Aachen University, Aachen, Germany

${ }^{2}$ Department of Orthopedic Trauma Surgery, Ningbo No.6 Hospital, Ningbo, China

${ }^{3}$ Department of Orthopedic Trauma Surgery, The Second Affiliated Hospital and Yuying Children's Hospital of Wenzhou Medical University, Wenzhou, China
${ }^{4}$ Institute of Anatomy and Cell Biology, Saarland University, Saarbrücken, Germany

${ }^{5}$ To whom correspondence should be addressed at Department of Orthopedic Trauma Surgery, Ningbo No.6 Hospital, Ningbo, China. E-mail: Xuding831129@126.com 
affected by MALP-2 treatment, the lung tissue presented with significant alterations. Reduced MPO activity was lowest in group ECLP (ECLP 11,196.77 $\pm 547.81 v s$. ETH 12,773.94 \pm 1011.76; $p=0.023$ resp. vs. 6CLP $13,155.19 \pm 423.99, p=0.016$ ) in experimental groups. Also, histological damage after MALP-2 application was lowest in ECLP animals (ECLP $0.50 \pm 0.08$ vs. ETH $0.71 \pm 0.05, p=0.034$ resp. vs. 6CLP $0.64 \pm 0.08, p=0.021$ ). Furthermore, MALP-2 treatment was associated with a trend towards improved survival in the ECLP group (ECLP 83.3\% vs. ETH 66.7 and 6CLP 58.3\%, $p>0.05$ ). Based on our results, MALP-2 might have beneficial effects on the clinical course after hemorrhage and sepsis by reducing pulmonary damage and PMN infiltration. This might also affect survival. According to our data, MALP-2 should be given at the earliest possible time point after the onset of sepsis. However, the optimal dosage and confirmation of our results in larger cohorts need to be the focus of further research.

KEY WORDS: MALP-2; Murine two-hit model; Hemorrhagic shock; Sepsis; Inflammatory response.

\section{INTRODUCTION}

Trauma represents the leading cause of death in patients under 40 years of age [1]. Especially in the later posttraumatic course, adverse outcome is related to a dysfunctional immune response that predisposes to infectious complications (e.g., sepsis) and organ dysfunction (adult respiratory distress syndrome, ARDS) [2, 3]. In this context, the lung is a primary target organ for inflammationassociated tissue damage due to high exposition to inflammatory mediators and immune cells that pass the lung via the bloodstream [4]. Thus, target-orientated treatment options for beneficial modulation of the posttraumatic immune response are of utmost importance to reduce infectious complications and severe organ damage $[5,6]$.

Toll-like receptors (TLRs) represent major therapeutic targets as they can recognize microorganisms that invade the body and activate immune cell responses [7]. TLRs initiate innate and adaptive immune responses via two cascades: the first one acts by the main adaptor protein, myeloid differentiation factor 88 (MyD88), the second via the adaptor TIR-domain-containing adaptor-inducing interferon- $\beta$ (TRIF), also known as the MyD88independent pathway. As one of the key factors for posttraumatic activation of the immune response TLR4 can signal through both aforementioned pathways in a timedependent manner [8]. Due to this important role of TLRs, direct modulation by a receptor agonist, either by (a) blocking the binding of TLR ligands to the receptor or (b) interfering the intracellular signaling pathways to stop the signal transduction could be a promising approach for the treatment of post-traumatic immune dysregulation, thereby reducing the incidence of infectious complications or organ damage [8].

As an agonist of Toll-like receptors, MALP-2 might therefore have great potential for clinical applications. So far, the relevance of MALP-2 treatment has only been investigated after isolated insults. Pfeifer et al. found that pre-treatment with MALP-2 resulted in a significant decrease in systemic IL-6 levels in a murine model of isolated hemorrhagic shock [9]. Zeckey et al. demonstrated that MALP-2 could improve survival and reduce systemic cytokine levels in a murine CLP model [10]. These results indicate the potential of MALP-2 to modulate the immune response thereby preventing complications and improving mortality rates.

In this study, we aimed to investigate whether MALP2 also exerts beneficial effects on the systemic inflammatory response and pulmonary immune response and damage in a clinically relevant two-hit model of TH and subsequent sepsis. In addition, the impact of the time point of MALP-2 application was assessed to elucidate the optimal moment starting MALP-2 treatment to potentially affect the outcome in a positive way.

We hypothesize that

1 The application of MALP-2 has a significant effect on systematic inflammation in the two-hit model of hemorrhagic shock and subsequent sepsis (end of trauma/hemorrhage and after CLP induction).

2 MALP-treatment reduces the incidence of pulmonary organ damage.

3 The effects of MALP-2 depend on the time point of application (end of trauma/hemorrhage vs. after CLP induction). 


\section{MATERIALS AND METHODS}

\section{Animal Care and Group Distribution}

To reduce the number of animals, data were gained from a larger project that was approved and registered by the Governmental Animal Care and Use Office (Landesamt für Natur, Umwelt und Verbraucherschutz Nordrhein-Westfalen, Recklinghausen, Germany, AZ: 84-02.04. 2013.A467). Male C57BL/6 mice, 8-10 weeks old and weighing $25 \pm 5 \mathrm{~g}$, were used for this study. Mice were divided into five different groups. The control group consisted of two sub-groups (control 1 and control 2, $n=$ 8/group) either undergoing TH and laparotomy (group 1) or TH and CLP (group 2). Based on the different injection times of MALP-2, we divided the mice into three experimental groups with 12 mice in each group (Table 1). Animals received a standard diet and tap water ad libitum. Mice were maintained in the animal research center with a 12 h light-dark cycle.

\section{General Anesthesia and Analgesia}

Before the experiments, anesthesia was induced using isoflurane (1.5-2\%, inhalation, Abbott Laboratories, Wiesbaden, Germany). To control pain, buprenorphine s.c. (Temgesic $囚$ ) was injected at a level of $0.05 \mathrm{mg} / \mathrm{kg}$ $30 \mathrm{~min}$ before instrumentation and thereafter was repeated every $8 \mathrm{~h}$. The absence of consciousness was proofed and documented by testing the reflex-status.

During instrumentation, induction of shock, and CLP, animals were placed in a supine position. They were kept warm by an underlying heating device. Additionally, heart frequency during shock was monitored by electrocardiography. All experimental procedures were performed under sterile conditions.

After terminating the experimental procedures $(\mathrm{TH}$ and CLP), animals were rewarmed and transferred to the prewarmed cage for continuous supervision. Analgesic therapy with buprenorphine was maintained three times a day as described above, and metamizole $(200 \mathrm{mg} / \mathrm{kg})$ was provided via the drinking water.

\section{Instrumentation and Pressure-Controlled Trauma- Hemorrhage}

A unilateral incision of approximately $1 \mathrm{~cm}$ in length was made in the area of the left femur to expose the femoral artery. The artery was temporarily ligated to prevent exsanguination during further instrumentation. Then, a small incision in the femoral artery was made using fine scissors to cannulate the vessel with a sterile plastic catheter (PE10). Next, the blood was slowly withdrawn using a digital blood pressure monitor (TSE Systems, BadHomburg, Germany) until shock state (mean arterial blood pressure (MAP) of $35 \mathrm{mmHg}$ ) was reached. This state was maintained for $90 \mathrm{~min}$. Thereafter, the withdrawn blood, as well as an equal amount of warmed saline solution were reinfused. This re-infusion was performed within $10 \mathrm{~min}$. Subsequently, the arterial catheter was removed, the vessel was closed with a ligature and the skin was sutured with a 4-0 suture. Afterward, animals were rewarmed and monitored as described above.

\section{Cecal Ligation and Puncture}

Cecal ligation and puncture (CLP) was performed $48 \mathrm{~h}$ after the application of TH except for animals that belong to control group 1. Animals from this group only received a laparotomy. After laparotomy, the cecum was carefully exposed and ligated as previously described [11]. Then, the cecum was punctured with a 21-gage needle and the cecal content was pressed out. After returning the cecum to its original position, $1 \mathrm{ml}$ of warmed $0.9 \% \mathrm{NaCl}$ solution was injected into the peritoneum and the abdominal layer was sutured. Afterward, animals were rewarmed and monitored as described above.

Table 1. The Specific Grouping of the Experiment

\begin{tabular}{lll}
\hline Groups & Treatment & \\
\hline Control 1 & TH, laparotomy, and vehicle administration & $n=8$ \\
Control 2 & TH, CLP, and vehicle administration & $n=8 \quad n=12 \quad n=12 \quad n=12 \quad$ TH, CLP, and MALP-2 application at the end of TH \\
ETH & TH, CLP, and MALP-2 application at 30 min. after CLP induction \\
ECLP & TH, CLP, and MALP-2 application at 6 h after CLP induction \\
6CLP & & $n$
\end{tabular}

$T H$, traumatic hemorrhage; $C L P$, cecal ligation and puncture; $M A L P-2$, Macrophage-activating lipopeptide-2; ETH, injection of MALP-2 at the end of TH; $E C L P$, injection of MALP-2 at $30 \mathrm{~min}$. after the end of CLP; $6 C L P$, injection of MALP-2 at $6 \mathrm{~h}$ after CLP 


\section{MALP-2 Application}

MALP-2 $(25 \mu \mathrm{g} / \mathrm{kg})$ or vehicle ( $0.9 \%$ sterile saline) were intraperitoneally administered at different time points over the course of the experiment according to the aforementioned group distribution (Table 1).

\section{Termination of the Experiment and Sample Collection}

The experiment ended $168 \mathrm{~h}$ after induction of $\mathrm{TH}$ $(120 \mathrm{~h}$ after CLP) or if defined ethical endpoints as published by Nemzek et al. forced the termination of the experiment [12]. Animals that died during the experiment were not included in the analysis while those that were in need to be euthanized before the end of the observation period were included in the analysis. The animals were exsanguinated by retrobulbar blood withdrawal. Blood samples were collected and immediately centrifuged at $3000 \mathrm{~g}$ for $5 \mathrm{~min}$ at $4{ }^{\circ} \mathrm{C}$. Plasma was frozen at $-80{ }^{\circ} \mathrm{C}$ until further analysis.

\section{Analysis of Cytokine Levels}

TNF- $\alpha$, IL-6, MIP- $1 \alpha$, IFN- $\gamma$, and IL-10 were evaluated using mouse premixed multi-analyte kits (R\&D System Inc., Minneapolis, MN, USA) according to the manufacturer's manual.

\section{Pulmonary Bacterial Clearance}

The left lung was rinsed three times with $1 \mathrm{ml}$ of PBS to obtain bronchoalveolar fluid (BALF). The collected pulmonary lavage fluid was immediately centrifuged at $400 \mathrm{~g}$ for $5 \mathrm{~min}$. The number of live bacteria was determined by the incubation of the pulmonary lavage $(95 \mu \mathrm{l})$ with $1 \%$ Triton X-100 (5 $\mu \mathrm{l})$ for $10 \mathrm{~min}$ for the lysis of the cells. Thereafter, serial dilutions were incubated on agar plates for $24 \mathrm{~h}$ at $37^{\circ} \mathrm{C}$. Bacterial colonies were counted with colony counting software [13]. The difference in bacterial clearance between each group was determined by comparing the number of bacterial colonies.

\section{Analysis of Myeloperoxidase Activity}

The right lung was then divided into two parts with one part being used for MPO determination via homogenization in a lysis buffer according to the manufacturer's protocol. Then, the homogenate was centrifuged at $12000 \mathrm{~g}$ for $15 \mathrm{~min}$ and the supernatant was collected for myeloperoxidase (MPO) activity assay. MPO-enzyme-linked immunosorbent assay kits (MPO ELISA kit, Hycultec GmbH
Beutelsbach, Germany) were used to quantify the MPO activity in lung tissues.

\section{Histology}

The other part of the right lung was filled with formalin and preserved for histological evaluation. Microscopically lung injury (PMN in the alveolar and interstitial space, hyaline membranes, proteinaceous debris filling the air spaces, and alveolar septal thickening) was assessed and graded according to a lung injury scoring system [14]. The lung injury score was evaluated by two pathologists who were blinded to the study groups.

\section{Data Analysis}

Data were analyzed using SPSS version 20.0 software (SPSS Inc., Chicago, IL, USA). A normal distribution of all data was examined by both the Shapiro-Wilk test and the Kolmogorov-Smirnov test. If the data were normally distributed, the values were expressed as the mean \pm SEM of observations. The data were summarized as a median (interquartile range (IQR)) when they were abnormally distributed. Group comparisons were evaluated using an ANOVA for normally distributed variables. Group comparisons were evaluated using the rank-sum test for abnormal distribution variables. The Bonferroni correction was executed for the pairwise comparison. The KaplanMeier survival curves were analyzed by the log-rank test. A $p$-value $<0.05$ was considered significant.

\section{RESULTS}

\section{Bacterial Clearance}

Those of control group 2 presented with a significantly higher number of bacterial colonies compared with animals of control 1 ( $p=0.002$, Fig. 1). Group ECLP tended to have higher numbers of bacterial colonies than groups ETH and 6CLP; however, there was neither a significant difference between the experimental groups and control group 1 nor between the experimental groups themselves $(p>0.05$, Fig. 1).

\section{Pulmonary MPO Activity}

Pulmonary MPO activity was highest in control group 2 (Fig. 2), whereas it was lowest in control group 1. There were significant differences between the experimental groups and control group 1 (Fig. 2). MPO activity was 


\section{Number of bacterial colonies}

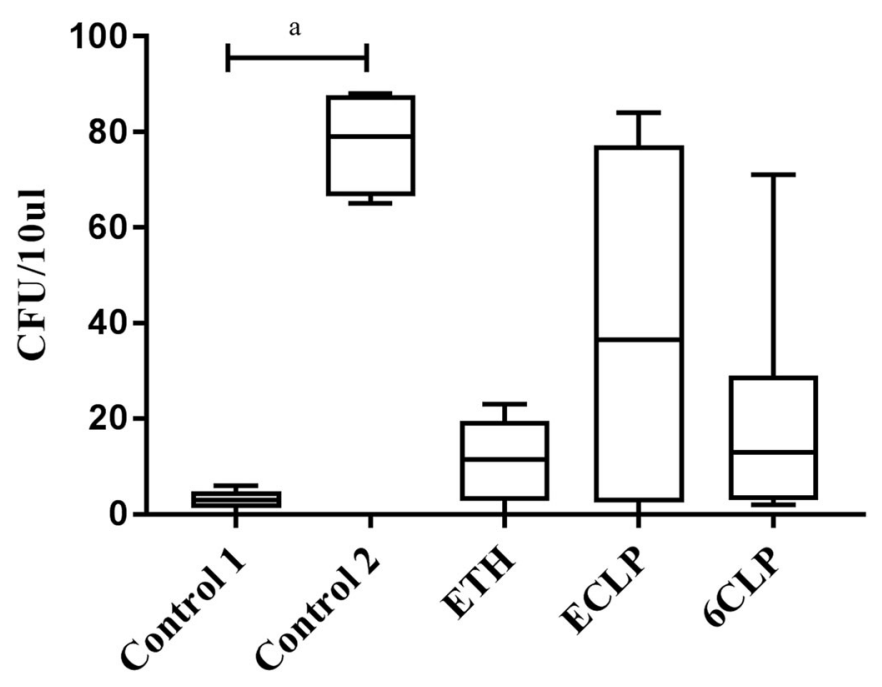

\section{Lung lavage fluid}

Fig. 1. The number of colonies after bacterial incubation in each group of lung lavage fluid. ${ }^{\text {a }}$ statistically significant difference between two groups $(p<0.05)$.

significantly lower in group ECLP than in groups ETH and 6CLP (ECLP 11,196.77 \pm 547.81 vs. ETH 12,773.94 \pm
1011.76, $p=0.023$ resp. vs. 6CLP 13,155.19 $\pm 423.99, p=$ 0.016) (Fig. 2).

\section{MPO Activity in the lung}

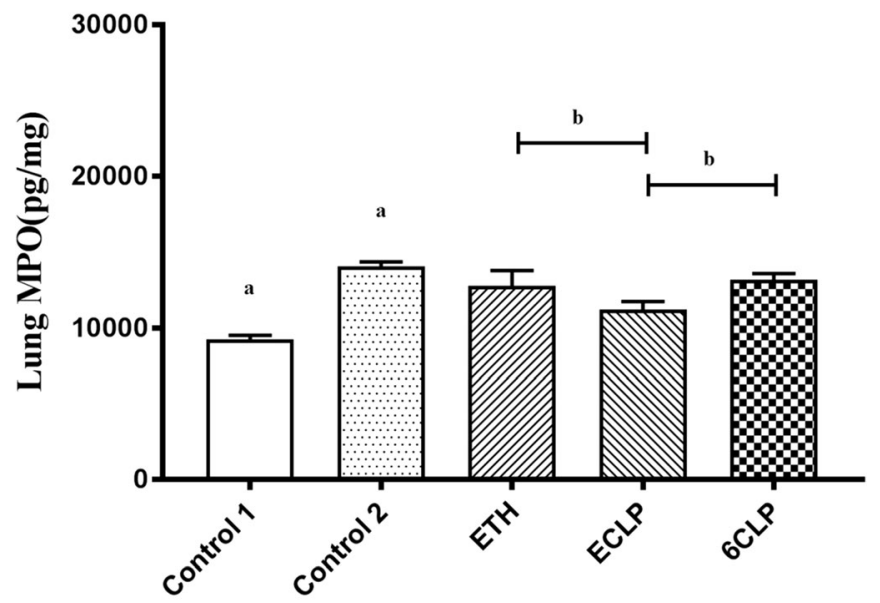

Groups

Fig. 2. The MPO activity of the lung in each group at the end of the experiment is shown in Fig. 2. ${ }^{\text { }}$ significant difference compared with the other groups $(p<$ 0.05 ). ${ }^{\text {bS }}$ tatistically significant difference between the two groups $(p<0.05)$ (TH: traumatic hemorrhage; CLP: cecal ligation and puncture; MALP-2: macrophage-activating lipopeptide-2; ETH: injection of MALP-2 at the end of TH; ECLP: injection of MALP-2 at 30 min. after the end of CLP; 6CLP: injection of MALP-2 at $6 \mathrm{~h}$ after CLP). 


\section{Histology of the Lung}

Control group 2 revealed the highest signs of tissue damage: its lung injury score was significantly higher compared with that of all other groups $(p=0.014)$. In the experimental groups, the lung injury score of the ECLP group was significantly lower than those of the ETH and 6CLP groups (ECLP $0.50 \pm 0.08 v s$. ETH $0.71 \pm 0.05, p=$ 0.034 resp. vs. 6CLP $0.64 \pm 0.08, p=0.021$ ) (Table 2 and Picture 1).

\section{Systemic Inflammatory Response}

At the end of the experiment, plasma levels of IL-6 were not detectable in control group 1 and no statistical differences among control group $2(55.6 \mathrm{pg} . / \mathrm{ml} \pm 17.6)$ and the experimental groups (ETH $40.8 \mathrm{pg} . / \mathrm{ml} \pm 13.1$, ECLP $56.0 \mathrm{pg} . / \mathrm{ml} \pm 29.2$, 6CLP $49.4 \mathrm{pg} . / \mathrm{ml} \pm 8.5$ ) was found (Fig. 3A). Compared with control group $1(0.16 \mathrm{pg} . / \mathrm{ml} \pm$ $0.11)$, the level of TNF- $\alpha$ in the ECLP group (2.27 pg. $/ \mathrm{ml}$ \pm 1.39 ) showed a significant increase ( $p=0.021$, Fig. 3B). In contrast, levels of IFN- $\gamma$ have significantly reduced in groups ETH $(1.77 \mathrm{pg} . / \mathrm{ml} \pm 4.34)(p=0.026)$, and 6CLP $(1.83 \mathrm{pg} . / \mathrm{ml} \pm 4.49)(p=0.014)$ compared with control group 1 ( $8.92 \mathrm{pg} . / \mathrm{ml} \pm 4.38$ ) (Fig. 3C). The expression of MIP- $1 \alpha$ showed no significant differences between all groups (Fig. 3D). In regard to anti-inflammatory IL-10 plasma levels were not detectable in group control 1 (Fig. 4). However, control group $2(4.79 \mathrm{pg} . / \mathrm{ml} \pm 5.13)$ and ECLP $(8.71 \mathrm{pg} . / \mathrm{ml} \pm 6.43)$ showed highest values. Yet, there was no significant difference between the groups.

\section{Survival Rate}

All mice survived in the control group 1. Four mice survived in control group 2. The group ETH has eight surviving mice. And ten and seven surviving mice were

Table 2. The Score of Each Group According to Lung Injury Scoring System

\begin{tabular}{ll}
\hline Groups & Lung injury score \\
\hline Control 1 & $0.20 \pm 0.08^{\mathrm{a}}$ \\
Control 2 & $0.86 \pm 0.05^{\mathrm{b}}$ \\
ETH & $0.71 \pm 0.05^{\mathrm{c}}$ \\
ECLP & $0.50 \pm 0.08^{\mathrm{d}}$ \\
6CLP & $0.64 \pm 0.08$ \\
\hline
\end{tabular}

${ }^{a}$ Control 1 vs. control 2, ETH, ECLP, 6CLP

${ }^{b}$ Control 2 vs. control 1, ETH, ECLP, 6CLP

${ }^{c}$ ETH vs. ECLP

${ }^{d}$ ECLP vs. 6 CLP

Statistical significance $p=<0.05$ in groups ECLP and 6CLP. There was a significant difference between control group $1(100 \%)$ and control group 2 $(50 \%)(p=0.025)$ and a clear trend toward an improved survival in group ECLP (88.3\%) compared with ETH (66.7\%) and 6CLP (58.3\%) animals was observed (Fig. 5).

\section{DISCUSSION}

The lung represents a major primary but also secondary target organ in case of trauma followed by sepsis. Pulmonary complications negatively influence the outcome after severe trauma and lead to extended ventilation rates, prolonged ICU-, and hospital stay as well as increased mortality rates [15]. Therefore, the prevention of lung damage and associated dysfunction by modulation of the posttraumatic immune response represents a potential therapeutic approach.

In our clinically relevant two-hit model, we found the following main results:

1 Infectious complications result in a significant aggravation of trauma-associated lung damage associated with high numbers of bacterial colonies

2 Application of MALP-2 resulted in lower sings of lung damage, reduced pulmonary bacterial load, and led toward lower mortality.

3 MALP-2 application was particularly beneficial if administered at the earliest possible stage after the onset of sepsis (ECLP) with

a reduced pulmonary tissue damage in histology analysis

b highest survival rates among of all groups with combines TH and CLP

\section{Immune Function and Organ Damage of the Lung}

The number of bacterial cultures found in BALF of control group 1 was significantly lower than that in control group 2. These findings are in line with other studies investigating the bacterial burden after $\mathrm{TH}$ versus sepsis and underline the validity of the used model $[16,17]$. However, the bacterial burden in MALP-treated groups tended to present with lower levels than animals with vehicle application after TH and CLP. This finding supports results presented by Reppe et al. who performed bacterial clearance experiments at $24 \mathrm{~h}, 48 \mathrm{~h}$, and $72 \mathrm{~h}$ after MALP-2 intervention in their mouse model of pneumonia [18]. It was found that there was no significantly 


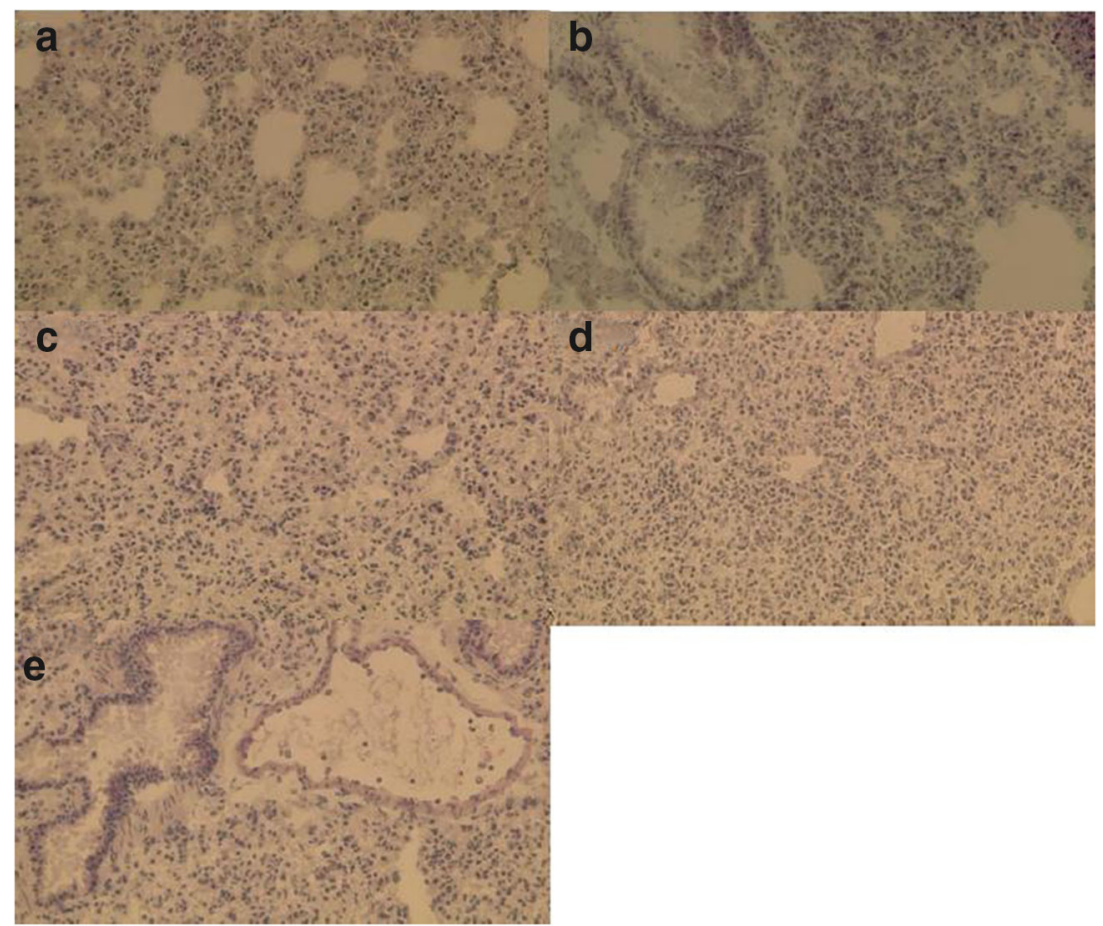

Picture 1. a-e Histopathology of infected lung tissue from mice $(\times 40)$. Figure $2 \mathrm{~A}$, the typical lung tissue from the group control 1. Figure 2B, the typical lung tissue from group control 2. Figure 2C, the typical lung tissue from the group of ETH. Figure 2D, the typical lung tissue from the ECLP group. Figure $2 \mathrm{E}$, the typical lung tissue from the 6CLP group. Figure 2A shows typical lung tissue sections in control group 1 with a clear tissue structure, thin alveolar walls, and no relevant PMN infiltration. Figure 2B shows typical lung tissue sections in control group 2 with inflammatory cell infiltration and the loss of lung tissue structure. Figure $2 \mathrm{C}$ represents the lung tissue section of the ETH group. Figure 2D demonstrates lung tissue damage and edema in the ECLP group. Figure 2E shows typical lung tissue sections of the 6CLP group. From Fig. 2B-E, the sections show varying degrees of inflammatory cell infiltration and exudation, loss of lung tissue structure, interstitial edema, and hemorrhage and the disappearance of the normal alveolar structure.

improved clearance of lung bacteria in the corresponding MALP-2 treatment group relative to the control group. In a follow-up study, Reppe et al. more specifically described a compartment-specific effect of MALP-2 treatment in the lung. In this context, a lowered number of bacterial colonies in the perivascular and in the peribronchial compartment was found, whereas numbers stayed persistently high in the pleural areas $[18,19]$. The authors explained this confined effect of MALP-2 by the unique pulmonary environment, with specialized peribronchial capillaries enabling improved pathogen clearance via rapidly migrating macrophages [20]. As the high pleural bacterial numbers however were unaffected by MALP-2 treatment, total lung bacterial load remained unaffected and the bacterial burden in the entire lungs was not significantly reduced by MALP2 [18]. Also, results from Zeckey et al. from a murine sepsis model might support a protective role of MALP-2 for lung tissue. While the significantly reduced levels of IL-6, TNF- $\alpha$, and MIP- $1 \alpha$ in the alveolar tissue found at the end of the observation period (96 h) might be interpreted as a residual pulmonary response on the one hand [10], Jörgens et al. showed that local IL-10 in the lung was significantly increased after pre-treatment of alveolar macrophages with MALP-2 [21] on the other hand. Thus, local pre-treatment with MALP-2 might beneficially influence the lung against inflammation by elevated antiinflammatory IL-10 concentrations and lowered proinflammatory mediator levels. However, as the experimental setups as well as observation times were different and the application mode of MALP-2 (aerosol vs. peritoneal injections) varied, future studies should focus on the impact of MALP-2 on systemically active inflammatory mediators that potentially harm the lung as a secondary target to improve outcome in critically ill patients.

MPO is the most abundant protein component expressed by PMN. Thus, MPO activity is one of the earliest signs in regard to neutrophil infiltration [22]. The present study revealed that an additional infectious insult 

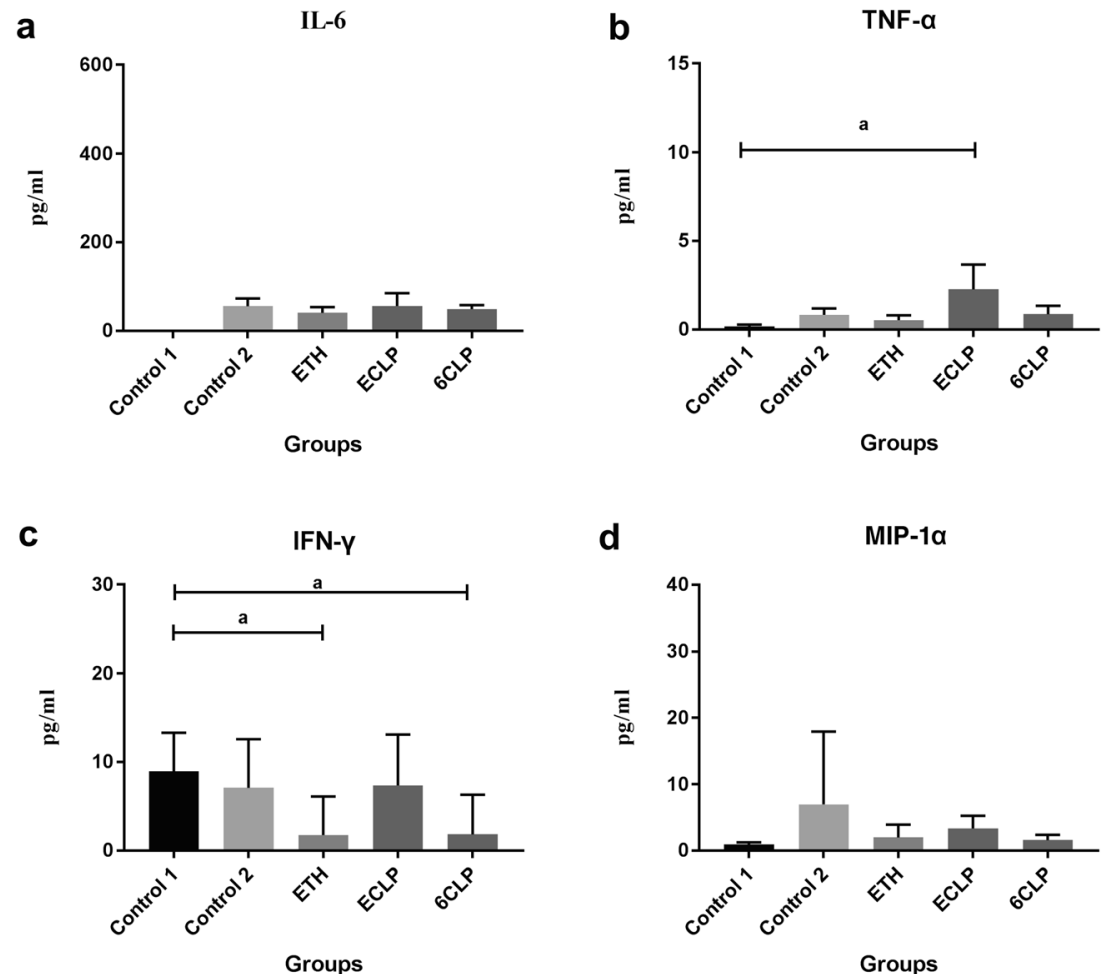

Fig. 3. a-d Expression levels of IL-6, TNF- $\alpha$, IFN- $\gamma$, and MIP- $1 \alpha$ at the end of the experiment. ${ }^{\text {a }}$ Statistically significant difference in two groups $\left({ }^{\mathrm{a}} p<0.05\right)$.

\section{IL-10}

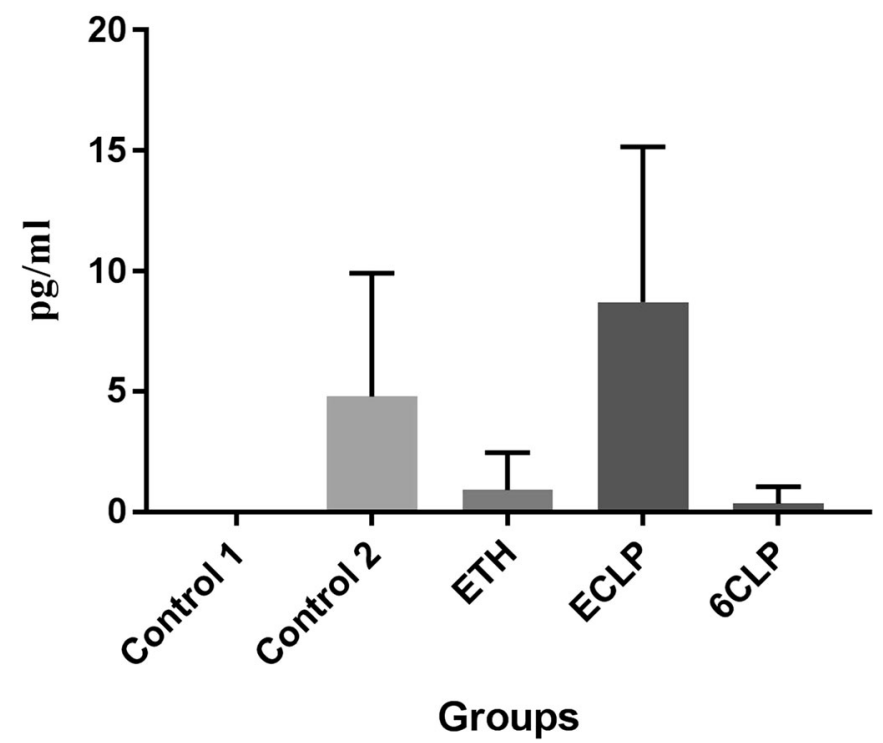

Fig. 4. Expression level of IL-10 at the end of experiment. 


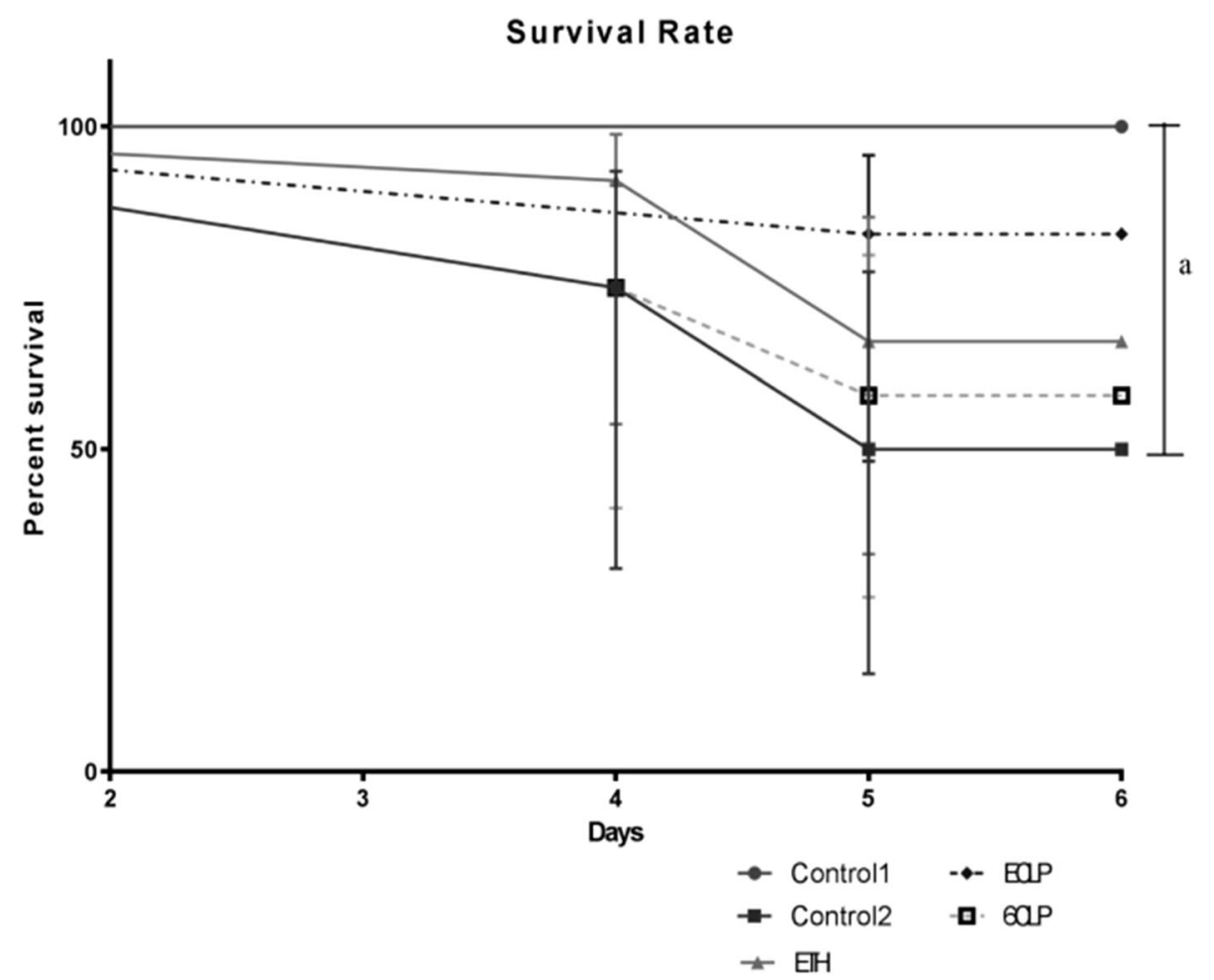

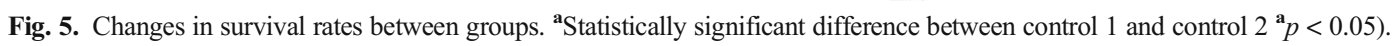

resulted in significantly increased pulmonary neutrophil infiltration reflected by enhanced MPO activity. However, MALP-2 application resulted in decreased values independent from the application time point compared with the two-hit group with vehicle treatment, indicating that MALP-2 has a significant inhibitory effect on the infiltration of PMN into the lung. In accordance, Zeckey et al. also found decreased MPO activity after applying MALP-2 in an isolated septic mouse model [10]. This finding and the aforementioned improvement of bacterial clearance might be assumed as a MALP-2-related augmentation and acceleration of the immune response with reduced MPO levels interpreted as a residuum after trauma followed by sepsis. This is even more likely as PMNs are known to survive only for $24 \mathrm{~h}$ after secretion and the observation time of the present model lasted for $168 \mathrm{~h}$ respectively $96 \mathrm{~h}$ in the model of Zeckey et al. [23]. Focusing on the best time point of MALP-2 application this is the first study to show that the time of MALP-2 intervention is best at the earliest time point after the development of sepsis. This provides a useful reference for further experimental studies and potential clinical applications in the future. However, literature regarding the aforementioned association between MALP-2 and MPO- activity is inconsistent. While some studies found that MALP-2 itself induces neutrophil infiltration into the lung and liver tissue [24, 25], Pfeifer et al. found that pretreatment with MALP-2 before the induction of shock did not have much of an effect on the MPO activity of the lung [9]. However, Pfeifer et al. terminated the experiment $6 \mathrm{~h}$ after the induction of shock. Therefore, the authors pointed out that further studies with a longer observation period and a more clinically relevant setup with the induction of second hits (additional surgery, infections) are required. Compared with the study presented by Pfeifer $e t$ al., we significantly extended the observation time and took the discussed limitations into account.

In accordance with MPO-levels, an additional infectious insult resulted in an aggravation of histological signs of lung damage. Intervention with MALP-2 alleviated local organ damage to the lungs. Our findings confirm the results that were reported earlier [18]. In lung tissue sections that were taken $48 \mathrm{~h}$ after pneumococcal infection, it was found that pneumococci were almost absent around the bronchi and pulmonary blood vessels in MALP-2 treated groups compared with the untreated pneumonia mice. In a follow-up study, MALP-2 was applied to investigate its influence on a secondary infection with 
S. pneumoniae after influenza virus infection [19]. It was found that treatment with MALP-2 significantly altered the accumulation of PMN in the interstitial lung, bronchi, and alveoli. These findings are consistent with the results of the present study. The three experimental groups (ETH, ECLP, 6CLP) treated with MALP-2 showed beneficial effects in regard to typical signs of pulmonary organ damage associated with sepsis. As for MPO-levels, MALP-2 application at the earliest time point after development of sepsis (group ECLP) also presented with the lowest lung injury score. In conclusion, the current data suggest an improved impact of MALP-2 in regard to lung damage. Especially the application of MALP-2 at the earliest onset of sepsis indicates a protective effect on the lung and therefore seems to be most promising.

\section{Systemic Inflammation}

By the end of the experiment, it was found that the MALP-2 application had no influence on systemic proinflammatory cytokine (IL-6, TNF- $\alpha$, and IFN- $\gamma$ ) and chemokine (MIP- $1 \alpha$ ) levels compared with vehicle administration. Thus, our findings are in line with data published by Zeckey et al. who also did not observe a significant impact of MALP-2 on pro-inflammatory mediator levels (IL-6, TNF- $\alpha$, and MIP- $1 \alpha$ ) $96 \mathrm{~h}$ after induction of isolated sepsis [10]. In a murine model of isolated TH, Pfeifer et al. found that MALP-2 application before the insult resulted in a more balanced migration of PMN into different body compartments instead of accumulating in one [9]. Based on this finding, it might be speculated that a similar phenomenon is also applicable for immunocompetent cells after the MALP-2 application. Possibly, e.g. macrophages localized in a specific organ system are stimulated by MALP- 2 to migrate into the circulation, thereby increasing systemic pro-inflammatory mediator levels. However, this macrophage migration might also have contributed to the MALP2-associated reduction of pulmonary damage, as decreased numbers of macrophages in the lung might be associated with reduced inflammation-related organ lesions.

Regarding the systemic anti-inflammatory factor IL10 , the experimental groups did not show any statistically significant differences from the control groups. Thus, observations reported by Pfeifer et al. were confirmed [9]. Also, Feterowski et al. described no elevation of IL-10 after systemic injection of MALP-2 in mice. The authors found that the systemic levels of IL-10 were higher in Pam3Cys treated mice compared with MALP-2 treatment [26]. These differences in the kinetics of cytokine induction were explained by an upregulation of ST2, a negative regulator of TLR signaling. ST2 negatively regulates TLR4 and IL$1 \mathrm{R}$ signaling via the sequestration of MyD88. The upregulation of ST2 in MALP-2-treated animals was more pronounced compared with Pam3Cys treatment. Probably the impact of MALP-2 on TLR2/TLR6, therefore, is more profound compared with the activation of TLR2/TLR1 by Pam3Cys. It is conceivable that the upregulation of ST2 contributes to the suppression of cytokine induction in MALP-2-treated mice [26].

\section{Survival Rate}

The application of MALP-2 showed a clear trend toward a better survival rate. Consistent with reduced pulmonary organ damage, MALP-2 application at the onset of sepsis was associated with the highest survival rate. Also, previous studies found reduced mortality rates after MALP-2 application in murine models with severe infections (e.g. sepsis, pneumonia) $[10,18,26]$. Although not statistically significant, our study supports these previous findings. Yet, the different results of these studies might be affected by diverse factors, such as experimental setups and endpoints. Comparing different studies, we found that the dose of applied MALP-2 showed a wide range between 0,1 , and $1000 \mu \mathrm{g} / \mathrm{kg}[10,18,26]$. In regard to the current literature and due to its novel application in a two-hit model, a moderate dose of $25 \mu \mathrm{g} / \mathrm{kg}$ was used in the present study. Thus, it is very likely that the amount of applied MALP-2 plays a crucial role in regard to immunologic modulation and thus prevention of secondary complications. However, further studies are needed to focus on dose-finding and optimal timing of MALP-2 application in clinically realistic models with clear outcome parameters. As no comparable reports regarding MALP-2 application in two-hit models were found, our results may be a useful reference for future research.

\section{Strengths and Limitations}

For the first time, MALP-2 was applied to a murine "two-hit" model to observe its effects. The experimental set up mimicked clinical reality. At the same time, several restrictions from previous studies were considered; however, also the present model is not fully free from limitations. First of all, all procedures were performed with animals under general anesthesia, which might cause some modulation of cellular injury. Moreover, acute trauma in humans does not occur under the conditions described here. However, ethical guidelines for animals and a moral imperative do not permit any other approach. In addition, although aiming to provide a clinically realistic setup, the 
complex injury pattern of our model is influenced by many variables (e.g. drugs, infusions, diet, and sample collection) that may also affect the results. Lastly, data was gained only at the end of the observation time and blood bacterial clearance tests were not conducted as well as inflammatory cytokines and chemokines in the distal organ tissue should be analyzed in future studies.

\section{CONCLUSION}

In conclusion, MALP-2 seems to have beneficial effects on the clinical course after hemorrhage and sepsis by reducing pulmonary damage and PMNs infiltration. This might also positively affect survival. Our findings suggest that MALP-2 should be given at the earliest possible time point around the onset of sepsis. Further studies must focus on the effect of MALP-2 in regard to different organ systems and the optimal dosage should be defined in larger cohorts.

\section{REFERENCES}

1. Krug, E.G., G.K. Sharma, and R. Lozano. 2000. The global burden of injuries. American Journal of Public Health 90 (4): 523-526.

2. Cabrera, C.P., J. Manson, J.M. Shepherd, et al. 2017. Signatures of inflammation and impending multiple organ dysfunction in the hyperacutephase of trauma: a prospective cohort study. PLoS Medicine 14 (7): e1002352.

3. Lord, J.M., M.J. Midwinter, Y.F. Chen, A. Belli, K. Brohi, E.J. Kovacs, L. Koenderman, P. Kubes, and R.J. Lilford. 2014. The systemic immune response to trauma: an overview of pathophysiology and treatment. Lancet. 384 (9952): 1455-1465.

4. Perl, M., J. Lomas-Neira, F. Venet, C.S. Chung, and A. Ayala. 2011. Pathogenesis of indirect (secondary) acute lung injury. Expert Review of Respiratory Medicine 5 (1): 115-126.

5. Wadhwa, J., and R. Sood. 1997. Multiple organ dysfunction syndrome. National Medical Journal of India 10 (6): 277-282.

6. Masuda, K., and T. Kishimoto. 2018. A potential therapeutic target RNA-binding protein, arid5a for the treatment of inflammatory disease associated with aberrant cytokine expression. Current Pharmaceutical Design 24 (16): 1766-1771.

7. Mukherjee, S., S. Huda, and S.P. Sinha Babu. 2019. TLR polymorphism in host immune response to infectious diseases: a review. Scandinavian Journal of Immunology 3: e12771.

8. Gao, W., Y. Xiong, Q. Li, et al. 2017. Inhibition of toll-like receptor signaling as a promising therapy for inflammatory diseases: a journey from molecular to nano therapeutics. Frontiers in Physiology 19 (8): 508 .
9. Pfeifer, R., T. Tschernig, P. Lichte, D. Dombroski, P. Kobbe, and H.C. Pape. 2013. MALP-2 pre-treatment modulates systemic inflammation in hemorrhagic shock. Journal of Inflammation (Lond). 10 (1): 17.

10. Zeckey, C., T. Tschernig, F. Hildebrand, M. Frink, C. Frömke, M. Dorsch, C. Krettek, and T. Barkhausen. 2010. Macrophage activating lipopeptides-2 exerts protective effects in a murine model of sepsis. Shock. 33: 614-619.

11. Horst, K., J. Höfler, L. Martin, J. Greven, T. Schürholz, T.P. Simon, G. Marx, and F. Hildebrand. 2019. Geriatric polytraumacardiovascular and immunologic response in a murine two-hit model of trauma. The Journal of Surgical Research 241: 87-94.

12. Nemzek, J.A., H.Y. Xiao, A.E. Minard, G.L. Bolgos, and D.G. Remick. 2004. Humane endpoints in shock research. Shock. 21 (1): 17-25.

13. Tadié, J.M., H.B. Bae, S. Banerjee, J.W. Zmijewski, and E. Abraham. 2012. Differential activation of RAGE by HMGB1 modulates neutrophil-associated NADPH oxidase activity and bacterial killing. American Journal of Physiology. Cell Physiology 302 (1): C249C256.

14. Matute-Bello, G., G. Downey, B.B. Moore, S.D. Groshong, M.A. Matthay, A.S. Slutsky, W.M. Kuebler, and Acute Lung Injury in Animals Study Group. 2011. An official American Thoracic Society workshop report: features and measurements of experimental acute lung injury in animals. American Journal of Respiratory Cell and Molecular Biology 44: 725-738.

15. Eberhard, L.W., D.J. Morabito, M.A. Matthay, R.C. Mackersie, A.R. Campbell, J.D. Marks, J.A. Alonso, and J.F. Pittet. 2000. Initial severity of metabolic acidosis predicts the development of acute lung injury in severely traumatized patients. Critical Care Medicine 28 (1): $125-131$.

16. Kaner, Z., D.E. Ochayon, G. Shahaf, B.M. Baranovski, N. Bahar, M. Mizrahi, and E.C. Lewis. 2015. Acute phase protein $\alpha 1$ antitrypsin reduces the bacterial burden in mice by selective modulation of innate cell responses. The Journal of Infectious Diseases 211: 1489-1498.

17. Baxevanos, N., E.J. Giamarellos-Bourboulis, A. Pistiki, M. Korre, D.I. Droggiti, and T. Tsaganos. 2013. Bacterial translocation induces proinflammatory responses and is associated with early death in experimental severe injury. The Journal of Surgical Research 185: 844-850.

18. Reppe, K., T. Tschernig, A. Lührmann, V. van Laak, K. Grote, M.V. Zemlin, B. Gutbier, H.C. Müller, M. Kursar, H. Schütte, S. Rosseau, R. Pabst, N. Suttorp, and M. Witzenrath. 2009. Immunostimulation with macrophage-activating lipopeptide- 2 increased survival in murine pneumonia. American Journal of Respiratory Cell and Molecular Biology 40: 474-481.

19. Reppe, K., P. Radünzel, K. Dietert, T. Tschernig, T. Wolff, S. Hammerschmidt, A.D. Gruber, N. Suttorp, and M. Witzenrath. 2015. Pulmonary immunostimulation with MALP-2 in influenza virus-infected mice increases survival after pneumococcal superinfection. Infection and Immunity 83 (12): 4617-4629.

20. Pabst, R., and T. Tschernig. 2002. Perivascular capillaries in the lung: an important but neglected vascular bed in immune reactions? The Journal of Allergy and Clinical Immunology 110 (2): 209-214.

21. Jörgens, G., F.C. Bange, P.F. Mühlradt, R. Pabst, U.A. Maus, and T. Tschernig. 2009. Synthetic lipopeptide MALP-2 inhibits intracellular growth of Mycobacterium bovis BCG in alveolar macrophagespreliminary data. Inflammation. 32: 247-251. 
22. Nauseef, W.M. 2007. How human neutrophils kill and degrade microbes: an integrated view. Immunological Reviews 219: 88-102.

23. McCracken, J.M., and L.A. Allen. 2014. Regulation of human neutrophil apoptosis and lifespan in health and disease. Journal of Cell Death. 8 (7): 15-23.

24. Lührmann, A., U. Deiters, J. Skokowa, et al. 2002. In vivo effects of a synthetic 2-kilodalton macrophage-activating lipopeptide of Mycoplasma fermentans after pulmonary application. Infection and Immunity 70: 3785-3792.

25. Kaufmann, A., P.F. Mühlradt, D. Gemsa, et al. 1999. Induction of cytokines and chemokines in human monocytes by Mycoplasma fermentans-derived lipoprotein MALP-2. Infection and Immunity 67: 6303-6308.

26. Feterowski, C., A. Novotny, S. Kaiser-Moore, P.F. Mühlradt, T. Roßmann-Bloeck, M. Rump, B. Holzmann, and H. Weighardt. 2005. Attenuated pathogenesis of polymicrobial peritonitis in mice after TLR2 agonist pre-treatment involves ST2 up-regulation. International Immunology 17: 1035-1046.

Publisher's Note Springer Nature remains neutral with regard to jurisdictional claims in published maps and institutional affiliations. 